\title{
Complications and Clinical Outcome 18 Months After Bipolar and Monopolar Transurethral Resection of the Prostate
}

\author{
Tim Fagerstrom, Claes R. Nyman and Robert Hahn
}

\section{Linköping University Post Print}

N.B.: When citing this work, cite the original article.

Original Publication:

Tim Fagerstrom, Claes R. Nyman and Robert Hahn, Complications and Clinical Outcome 18 Months After Bipolar and Monopolar Transurethral Resection of the Prostate, 2011, Journal of endourology, (25), 6, 1043-1049.

http://dx.doi.org/10.1089/end.2010.0714

Copyright: Mary Ann Liebert, Inc. http://www.liebertpub.com/

Postprint available at: Linköping University Electronic Press http://urn.kb.se/resolve?urn=urn:nbn:se:liu:diva-69900 


\title{
Complications and Clinical Outcome 18 Months After Bipolar and Monopolar Transurethral Resection of the Prostate
}

\author{
Tim Fagerström, M.D., Claes R. Nyman, M.D., Ph.D., and Robert G. Hahn, M.D., Ph.D. ${ }^{1}$
}

\begin{abstract}
Purpose: To compare bipolar resection with the conventional monopolar transurethral resection of the prostate (TURP) with respect to peri- and postoperative complications and long-term outcome.

Patients and Methods: Patients with consecutive benign prostatic hyperplasia needing surgery $(n=185)$ from the hospital's waiting list were randomized to TURP using either a bipolar or a monopolar system. Peri- and postoperative parameters were monitored, complications were registered, and timed micturition/International Prostate Symptom Score (TM/IPSS) forms were collected at 3 and 6 weeks and at 6 and 18 months.

Results: Bipolar surgery was followed by a $16 \%$ to $20 \%$ higher percentage of the patients reporting ongoing improvement (fractional IPSS change $>2)$ at 3 and 6 weeks after the surgery $(p<0.05)$. There were fewer readmissions in the bipolar group than in the monopolar ( 5 vs. $13, p<0.05)$. No differences between the groups with respect to hospital stay and catheter duration was recorded. Bipolar and monopolar TURP resulted in marked and sustained improvements of IPSS, bother score, and TM.

Conclusions: Bipolar TURP, using the transurethral resection in saline (TURis) system, resulted in significantly fewer postoperative readmissions, faster postoperative recovery, and equally long-lasting good results in TM/ IPSS and bother score, as in monopolar TURP.
\end{abstract}

\section{Introduction}

$\mathbf{I}_{\mathrm{p}}^{\mathrm{N}}$ N THE PAST FEW YEARS the bipolar technique of resecting the prostate has become available worldwide ${ }^{1-3}$ and, currently, alongside other minimally invasive techniques, especially different laser modalities, challenges the monopolar transurethral resection of the prostate (TURP) as being the gold standard in treating benign prostatic hyperplasia (BPH). The high efficacy of TURP makes it one of the most common surgical procedures worldwide. In Sweden, approximately 6000 such operations are performed every year. However, TURP is rarely performed as day surgery, as peri- and postoperative complications may require special attention. ${ }^{4,5}$

Bipolar TURP has theoretical advantages that have to be further evaluated. A number of small studies with limited follow-up have been conducted without showing any clinically relevant differences. ${ }^{6-8}$ Our present study is one of the largest randomized studies with the longest follow-ups, using the transurethral resection in saline (TURis) system. The overall surgical hemorrhage during these bipolar operations was $34 \%$ smaller than during monopolar TURP, with the difference being $81 \%$ for the largest bleeders. ${ }^{9}$
In this study, we further explore the potential benefits of bipolar TURP by addressing perioperative fluid absorption, as well as the clinical outcome in terms of complications and patient satisfaction up to 18 months after surgery.

\section{Patients and Methods}

Patients

Between December 2005 and August 2008, 202 patients were allocated, using a random numbers table, to either TURP using a bipolar system or conventional monopolar technique. Inclusion criteria were a transrectal ultrasound prostatic volume of 30 to $100 \mathrm{~mL}$ and symptomatic BPH that required surgery because medical therapy had failed or urinary retention requiring an indwelling catheter. Patients with prostate volumes $<30 \mathrm{~mL}$ underwent transurethral incision of the prostate (TUIP), and those with volumes $>100 \mathrm{~mL}$ had open surgery according to hospital routine and European Association of Urology guidelines. ${ }^{10}$ Exclusion criteria were evident prostate or bladder cancer and core biopsy of the prostate within 3 months before surgery. Patients with signs of neurogenic bladder dysfunction were not

\footnotetext{
${ }^{1}$ Section of Urology, Södersjukhuset, Department of Clinical Science and Education, Karolinska Institutet, Stockholm, Sweden.

${ }^{2}$ Faculty of Health Sciences, Linköping University, Linköping, Sweden.
} 
enrolled in the study, neither were patients with urethral strictures. None of the patients had been subject to TURP before enrollment. The local Ethics Committee in Stockholm approved the study, and all participants gave written informed consent before surgery.

\section{Techniques}

Bipolar TURP was performed with the Olympus TURis system using the Olympus UES 40 generator (Tokyo, Japan) set at $280 / 100 \mathrm{~W}$ (cutting/coagulation). A 24 Charriere resectoscope with a $0.2 \mathrm{~mm} 12^{\circ}$ medium loop was employed for all resections. To prevent misleading resection weights, none of the operations was carried out with the vaporization option. Normal saline (0.9\% NaCl; Baxter, Kista, Sweden) was used as irrigant.

Monopolar TURP was performed with a Storz 24 Charriere resectoscope, using an ERBE ICC 350 generator (Tübingen, Germany) set at 130/50 W (cutting/coagulation mode). All resections were carried out with standard loops and factorymade irrigating fluid containing mannitol $3 \%$ and ethanol $1 \%$ (Baxter). ${ }^{11}$

To offer the same possibility for early detection and quantification of fluid absorption in both groups, ethanol was added to the saline to reach the same concentration as in the electrolyte-free irrigating fluid. Operations were terminated when uptake of irrigating fluid was indicated by presence of ethanol in the breath.

Treatment with anticoagulants was discontinued 3 (warfarin) and 7 days (salicylates) before surgery. Prothrombin time/international normalized ratio was measured before surgery, and a level of $\leq 1.6$ was accepted.

Antibiotic prophylaxis was given to patients with an indwelling catheter or other risk factor (i.e., bladder stone) on the day of surgery. Patients with a confirmed infection were treated with antibiotics for 7 to 10 days. The other patients received no antibiotics.

There was no selection of patients to any particular surgeon. Fourteen of the evaluable operations were performed by two residents, under continuous supervision of a specialist in the operating room, and the others by 10 specialists with at least 5 years' experience of urological surgery.

Seven patients in the monopolar group and nine patients in the bipolar group had general anesthesia. The remaining patients received spinal anesthesia.

Postoperative irrigation was turned off when the urine appeared to be clear. Our policy was to relieve the patient from the catheter in the first morning after surgery, provided that the irrigation had been terminated and urine was still clear.

\section{Measurements}

Operating time was taken as the period from the first cut in the prostate until a $20 \mathrm{~F}$ three-way hematuria catheter was inserted. The time required for additional procedures, such as cystolithotripsy, was not included.

The absorption of irrigating fluid was recorded. Every 10 minutes during surgery the end-expiratory ethanol concentration was measured with a hand-held Alcolmeter (Model SD-400; Lions Laboratories, S. Glamorgan, United Kingdom) and the volume of irrigant absorbed was estimated by using a regression equation based on 90 operations. ${ }^{11}$
Peri- and postoperative blood loss was measured with the Low Hemoglobin photometer method (HemoCue, Ängelholm, Sweden). ${ }^{12,13}$ The postoperative bleeding was assessed from end of surgery until the irrigation was switched off.

Recorded postoperative complications included infections and hemorrhage leading to re-admissions and prolonged hospital stay, as well as reoperations because of urethral or bladder neck strictures and incomplete resections.

Forms containing data on International Prostate Symptom Score (IPSS) / quality of life (QoL) and timed micturition (TM) were collected preoperatively (for patients without indwelling catheter) and then sent in by the patients at 3 and 6 weeks and at 6 and 18 months after surgery. Patients were reminded a maximum three times to return the forms. Patients with low IPSS (indicating obstruction) underwent cystoscopy to determine the incidence of urethral strictures.

\section{Statistical methods}

The Mann-Whitney $U$ test was used to evaluate numerical variables with a skewed distribution. Categorical variables were analyzed using $\chi^{2}$ test. Numerical data showing a skewed distribution were square-root-transformed before being tested by linear regression. Two-way analysis of variance was used, controlling for factors associated with absorption. $p<0.05$ was considered significant. Calculations were made in PASW Statistics 18.0.0 (SPSS, Inc., Chicago, IL).

\section{Results}

\section{Demographic data}

Baseline data were similar for the two groups, which comprised 185 evaluable patients. One-third of them had an indwelling catheter at the time of operation, and the others had a TM showing low-flow values as a sign of marked urinary outflow obstruction (Table 1 and Fig. 1).

\section{Perioperative measurements}

There were no statistically significant differences between bipolar and monopolar TURP with respect to the weight of resected tissue and operating time (Table 1). ${ }^{9}$ Absorption of irrigating fluid was detected by the ethanol method in 21 $(11 \%)$ of the patients, with a trend of fewer absorptions in the bipolar group, however, not statistically significant (Fig. 2). The absorptions occurred at the end of the procedure in all but one of these operations, and the duration of the absorption was at least 20 minutes in 10 of them. This resulted in premature termination of surgery in four patients in the monopolar group and in two patients in the bipolar group.

No obturator nerve affection was recorded in the two groups.

Absorption was symptomatic in three monopolar TURP patients. The patient with the largest absorption had a sudden drop in arterial pressure (from 150 to $90 \mathrm{~mm} \mathrm{Hg}$ systolic) at the end of surgery. This drop, combined with the observation that the absorbed volume exceeded $1 \mathrm{~L}$, led us to conclude the operation before excessive absorption occurred. This patient's serum sodium had decreased from 142 to $131 \mathrm{mmol} / \mathrm{L}$. Another patient complained about postoperative abdominal pain (absorption volume $807 \mathrm{~mL}$ ), and a third patient experienced headache (absorption volume $255 \mathrm{~mL}$ ). 
Table 1. Baseline, Perioperative, and Postoperative Data

\begin{tabular}{|c|c|c|c|}
\hline & \multicolumn{2}{|c|}{ Operation technique } & \multirow[b]{2}{*}{ Statistics, $\mathrm{p}$} \\
\hline & Bipolar & Monopolar & \\
\hline Age (year) & $69.5(7.2)$ & $72.7(8.4)$ & 0.007 \\
\hline Prostate volume $(\mathrm{mL})$ & $55.6(18.2)$ & $58.2(17.6)$ & ns \\
\hline IPSS preop (points) & $21.7(6.9)$ & $20.4(7.6)$ & ns \\
\hline Bother score preop (points) & $3.9(0.9)$ & $3.7(1.1)$ & ns \\
\hline Timed micturition preop (sec/1:st dl) & $24.5(14.4)$ & $27.7(17.9)$ & ns \\
\hline Operation time (min) & $62(23)$ & $66(23)$ & ns \\
\hline Resection weight (g) & $27.3(15.1)$ & $26.3(13.2)$ & ns \\
\hline Peroperative hemorrhage $(\mathrm{mL})$ & $235(24-1590)$ & $350(40-2500)$ & $<0.001$ \\
\hline Transfusions $(n)$ & 4 & 10 & $<0.01$ \\
\hline Preoperative catheter $(n)$ & 33 & 34 & ns \\
\hline Discharged with catheter $(n)$ & 8 & 10 & ns \\
\hline Catheter duration $(\mathrm{h})$ & $20(13-115)$ & $20(13-262)$ & ns \\
\hline Hospital stay (h) & $51(22-163)$ & $52(27-365)$ & ns \\
\hline
\end{tabular}

$n$, mean (SD), median (range) as appropriate.

IPSS = International Prostate Symptom Score; ns = not significant.

The incidence of fluid absorption, as well as monopolar surgery, was independently associated with a larger surgical hemorrhage (two-way analysis of variance based on lntransformed blood loss data; $p<0.02$ ). Operations with fluid absorption were also associated with a 15-minute longer operation time than the ones without fluid absorption $(p<0.02)$.

\section{Catheterization and hospitalization times}

If patients were nonfebrile and did no longer require postoperative bladder irrigation, catheter was removed at $06.00 \mathrm{a} . \mathrm{m}$. in the first postoperative morning, but re-inserted if bladder scan showed persistent urine retention of more than $200 \mathrm{~mL}$. One hundred thirty-one (71\%) catheters were terminated within 24 hours, and an additional 23 (12\%) patients had their catheters permanently removed within 48 hours with no difference between the groups. If a second try to get rid of the catheter did not succeed, patients were discharged with an indwelling catheter. A similar number of patients in the two groups were discharged with an indwelling catheter (Table 1). Only those with a successful catheter termination within the hospital stay are represented in the catheter time section in Table 1. The others are referred to as "discharged with catheter" (Table 1).

Of the total 18 patients discharged with indwelling catheter, only 1 (in the monopolar group) did not succeed to get rid of his catheter within the follow-up period. All the others could eventually terminate their catheterization (range 5-165 days after discharge) with no differences between the two techniques.

There were no overall differences in catheterization time or hospital stay (Table 1).

\section{Complications during follow-up/readmissions}

The number of readmissions was 14 for monopolar and 5 for bipolar ( $p<0.011$; Fig. 3). Readmissions were associated with larger hemorrhage per minute during the resection $(p<0.03)$. Two patients in the bipolar and one in the monopolar group were found to have urethral strictures in the follow-up. One of them had an internal urethrotomy after 4.5 months (bipolar), and the others were dilated once after 2 and 5.5 months, respectively. These three patients all presented persistent high IPSS and a feeling of weak stream and problem emptying the bladder, which led to investigation and intervention.

Six patients $(3 \%)$ were reoperated, two patients in the bipolar $(2 \%)$ and four $(5 \%)$ in the monopolar group. In addition to the single internal urethrotomy in the bipolar group, one patient underwent TUIP because of bladder neck stenosis. This occurred 14 months after the operation and was indicated by an increase in TM and bother scores. In the monopolar group, one TUIP and three re-TURPs were performed at 3.5 and 3, 4, and 12 months after the initial operation because of incomplete resections. The first of the re-TURPs had signs of absorption in the Alcolmeter, and thus only one lobe was resected. The second patient had a long operation time because of diffuse bleeding, and therefore surgery was prematurely terminated. The third patient also had a long operation time because of time-consuming lithotripsy initially, which in this case also led to an incomplete resection. All four of these patients could not terminate their catheterization and therefore were subject to further investigations leading to additional surgery.

Thirteen patients in both groups had infections that did not lead to readmission. A majority $(22 / 26)$ of the infections appeared within 24 hours after surgery, whereas the others became evident after 2 to 13 days. Of the patients with infections, 20 had preoperative risk factor such as indwelling catheter and/or bladder stone. Six patients without any risk factors developed infection.

\section{IPSS, bother score, and TM}

A detailed analysis of the IPSS forms showed that recovery occurred faster in the bipolar than in the monopolar group, since a larger fraction of the former patients reported ongoing improvement at both 3 and 6 weeks $(p<0.05$; Fig. 4 right). The difference was $20 \%$, at 6 weeks $(p<0.05)$. At 18 months the early differences between the groups had cancelled out. 

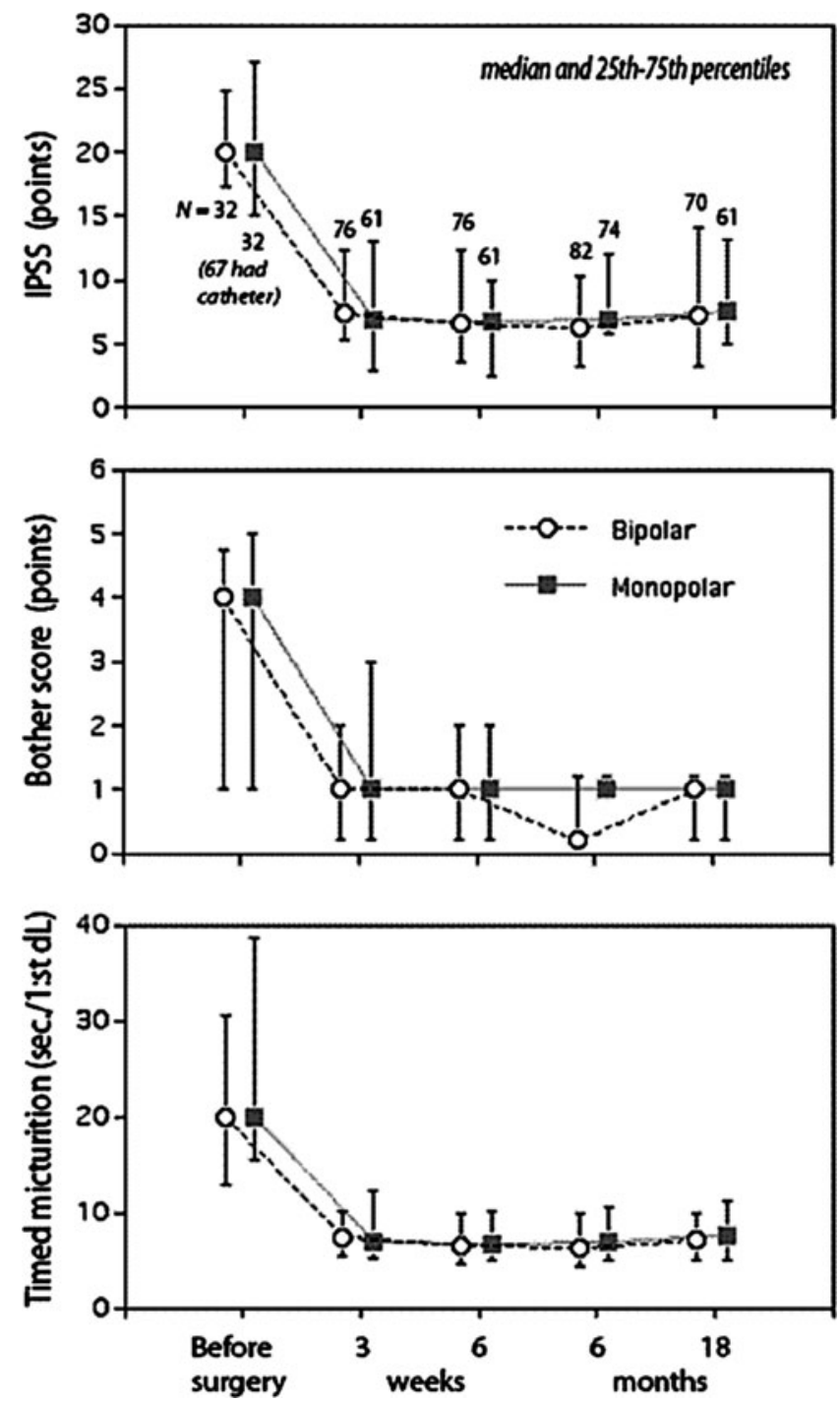

FIG. 1. Change in International Prostate Symptom Score (IPSS), quality of life, and timed micturition over time.

Both operating techniques markedly reduced the IPSS, bother score, and TM. The positive results remained throughout the follow-up period (Fig. 1). The presence of an indwelling catheter before surgery was associated with a lower IPSS at 6 weeks and 6 months $(p<0.05)$, but not at 18 months (stepwise multiple regression).

Patients with a preoperative indwelling catheter were not represented in the evaluation of the recovery at 3 weeks as the catheter precluded collection of meaningful baseline data. However, they do contribute to the total median at 3 weeks. The overall answering frequency was approximately $70 \%$ of eligible patients (i.e., not catheterized) at each evaluation point (Fig. 1).

\section{Discussion}

More patients operated with the bipolar technique reported early improvement in IPSS and QoL scores than those having monopolar surgery, and thus recovered faster. Bipolar TURP was followed by fewer readmissions, especially when caused by late hematuria. Both methods were associated with a

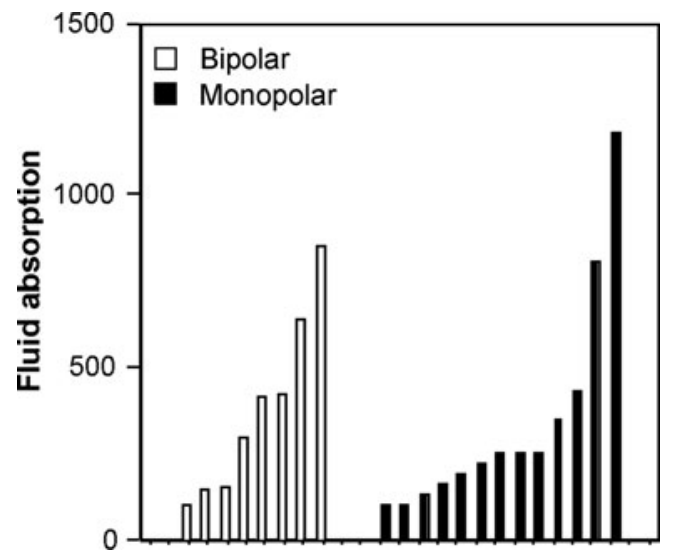

FIG. 2. The volume of irrigant absorbed for each patient in whom ethanol was detected in the exhaled breath. Each bar represents one patient.

similar incidence of fluid absorption. No differences were seen in catheterization time, associated infections, or hospital stay. As reported previously, the surgical hemorrhage and the transfusion rate were smaller during bipolar TURP, whereas the bleeding during postoperative irrigation was negligible after both types of surgery.

Bipolar and monopolar TURP both resulted in long-lasting improvement of symptoms associated to $\mathrm{BPH}$.

There are only a few previous randomized studies comparing the two techniques. Many of them are inconclusive, because of too small sample sizes. ${ }^{14,15} \mathrm{~A}$ mix of techniques are compared: vaporization versus resection ${ }^{16-18}$ and resection versus resection. ${ }^{19,20}$ Our $10 \%$ readmission rate is difficult to view in perspective, because readmissions have rarely been reported by previous authors. On the other hand, the observed reoperation rate of $3 \%$ is comparable to other studies, where the rates ranged between $0 \%$ and $9.6 \% .{ }^{16,17,21-23}$ The need for reoperation because of incomplete resection was lower in the bipolar group. This might partly be explained by the smaller perioperative bleeding, providing better visual control of the operating field. Our practice of not giving all patients antibiotics before surgery might explain why our incidence of infections is slightly higher than that reported by others. $^{22}$

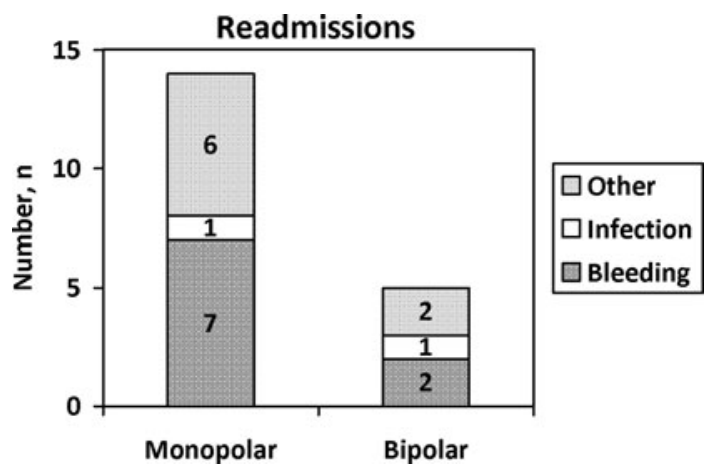

FIG. 3. The incidence of readmissions and their causes after transurethral resection of the prostate (TURP) using two different surgical techniques. "Others" comprised catheter problems, urge, and urinary retention. 

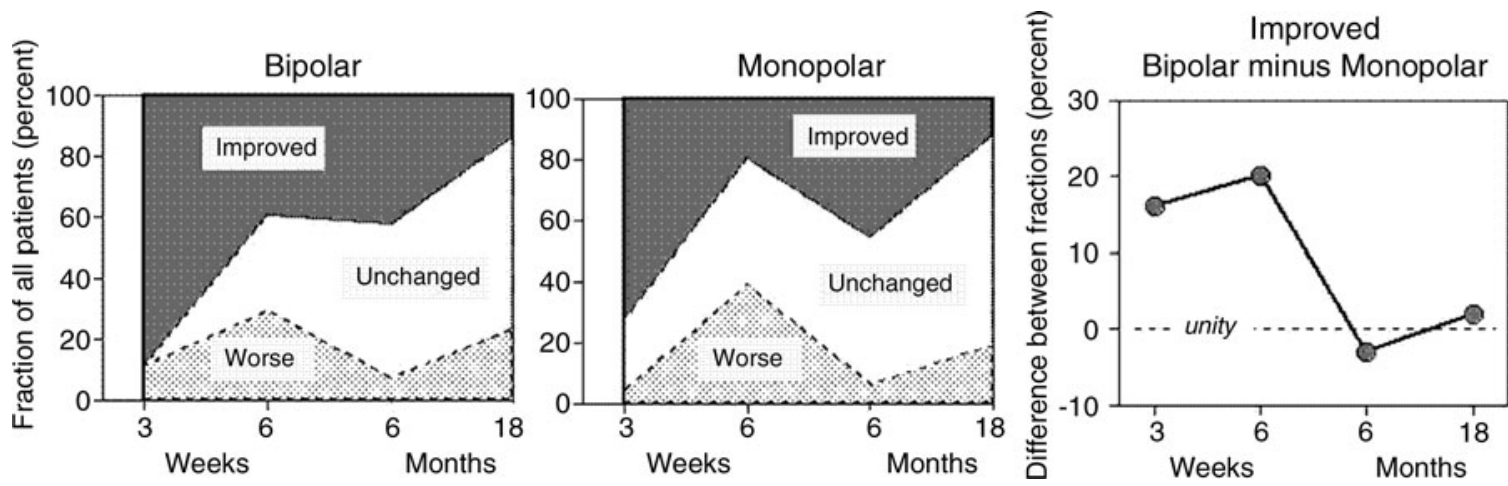

FIG. 4. The fraction of patients showing different trends of IPSS change after bipolar TURP (left) and monopolar TURP (middle). "Improved" is a decrease from the previous score by $>2$. "Unchanged" denotes a change of between -2 to +2 , and "Worse" is an increase by 2 . The right panel shows the difference in percentage of "Improved" between the groups.

Our median catheterization time of 20 hours is markedly shorter than in several earlier studies comparing the two techniques. $6,14,16,24$ This difference might be a matter of clinical routines and tradition, but may also reflect different interests between private and public caregivers. A long postoperative catheterization prolongs the hospital stay, greatly raising the cost of care. , $24^{2}$

There is little or no evidence supporting a need for prolonged postoperative catheter treatment, in particular because the median postoperative hemorrhage is very small. ${ }^{9}$

Fluid absorption is a complication often claimed to be eliminated by the use of bipolar TURP. ${ }^{25}$ In any event, measuring the decrease in serum sodium has no value for detecting the absorption during bipolar TURP, since the normal saline used for irrigation has a higher sodium concentration than serum. ${ }^{26}$ If the aim is to measure absorption, a marker such as ethanol should be used. As applied in the present study, the ethanol method revealed absorption events with approximately the same frequency, regardless of operating technique. Infusion studies in humans show that symptoms such as abdominal pain and slight confusion arise if more than $2 \mathrm{~L}$ of normal saline is infused intravenously. ${ }^{27,28}$ Larger amounts promote the development of pulmonary edema. ${ }^{29}$ Animal studies show that overload with normal saline is associated with tissue damage ${ }^{30}$ and a definite death, ${ }^{31}$ although it is less harmful than glycine $1.5 \%$. In turn, mannitol $3 \%$ is followed by fewer symptoms than absorption of glycine $1.5 \%$ in TURP patients. ${ }^{5}$

Bipolar surgery did offer benefits to the patients during surgery and the first 6 postoperative weeks. These included a smaller surgical hemorrhage ${ }^{9}$ and higher speed of recovery, the latter being indicated by our individual-based review of the IPSS forms, as well as by the fewer readmissions. Our long-term data confirm that both the bipolar and monopolar techniques of performing TURP improve urinary flow and reduce the IPSS and QoL scores. If a significant amount of prostatic tissue is removed, one could expect symptom relief regardless of technique. There might be little need for a follow-up longer than 18 months, since considerable data are available on monopolar surgery.

There were three patients who developed urethral strictures that necessitated surgery or dilatation. This number is too low to allow comparison between the operating techniques. However, the incidence is relatively low in comparison to earlier bipolar studies, in which figures up to $6.6 \%{ }^{14}$ have been reported. This difference might be explained by our short postoperative catheter time and by the smaller diameter of our resectoscopes, whereas our operating times were not on the short side, averaging 62 (bipolar) and 66 (monopolar) min. ${ }^{9}$ There is a debate about whether bipolar TURP is associated with a higher incidence of urethral strictures, ${ }^{14}$ whereas several studies suggest that there might not be a real difference between the two methods. ${ }^{2,15,32}$

Strict clinical routines were adhered to throughout the study, as narrow instruments as possible were used and the operation time was kept to a minimum. The postoperative catheterization was ended as soon as possible. Antibiotics were not prescribed routinely, which certainly contributed to the relatively high incidence of infections. This practice has been changed in our clinic based on the present results. Further, TURis has replaced monopolar TURP as the standard surgical treatment of $\mathrm{BPH}$.

A limitation of the study is that the cohort is too small to be subject to meaningful analyses of subgroups. Another potential limitation is that we wanted to study the urological "every-day" situation where the endoscopic operations are carried out by a heterogeneous group of surgeons. However, our resident team obtained similar results regarding surgical efficiency and postoperative outcome as in previous studies that included operations performed only by highly experienced urologists. ${ }^{1-3}$ Nevertheless, the present result can possibly be improved in studies where a very small group of highly drilled resectionists perform all operations.

\section{Conclusion}

The incidence of postoperative readmissions was significantly reduced and the postoperative recovery was faster in bipolar versus monopolar TURP. These benefits add to those of a previous report showing that the bipolar technique significantly decreases the perioperative blood loss. Both bipolar and monopolar resection showed good 
and long-lasting improvements of TM/IPSS and bother score.

\section{Disclosure Statement}

No competing financial interests exist.

\section{References}

1. Ho H, Yip S, Foo KT. Bipolar transurethral resection of prostate in saline: Preliminary report on clinical efficacy and safety at 1 year. J Endourol 2006;20:244-247.

2. Chen Q, Zhang L, Fan QL, et al. Bipolar transurethral resection in saline vs traditional monopolar resection of the prostate: Results of a randomized trial with a 2-year followup. BJU Int 2010;106:1339-1343.

3. Seckiner I, Yesilli C, Akduman B, et al. A prospective randomized study for comparing bipolar plasmakinetic resection of the prostate with standard TURP. Urol Int 2006;76: 139-143.

4. Ekengren J, Hahn RG. Blood loss during transurethral resection of the prostate as measured with the HemoCue photometer. Scand J Urol Nephrol 1993;27:501-507.

5. Hahn RG, Sandfeldt L, Nyman CR. Double-blind randomized study of symptoms associated with absorption of glycine $1.5 \%$ or mannitol $3 \%$ during transurethral resection of the prostate. J Urol 1998;160:397-401.

6. Singh H, Desai MR, Shrivastav P, et al. Bipolar versus monopolar transurethral resection of prostate: Randomized controlled study. J Endourol 2005;19:333-338.

7. Starkman JS, Santucci RA. Comparison of bipolar transurethral resection of the prostate with standard transurethral prostatectomy: Shorter stay, earlier catheter removal and fewer complications. BJU Int 2005;95:69-71.

8. Yang S, Lin WC, Chang HK, et al. Gyrus plasmasect: Is it better than monopolar transurethral resection of prostate? Urol Int 2004;73:258-261.

9. Fagerström T, Nyman CR, Hahn RG. Bipolar transurethral resection of the prostate causes less bleeding than the monopolar technique: A single-centre randomized trial of 202 patients. BJU Int 2010;105:1560-1564.

10. Madersbacher S, Alivizatos G, Nordling J, et al. EAU 2004 guidelines on assessment, therapy and follow-up of men with lower urinary tract symptoms suggestive of benign prostatic obstruction (BPH guidelines). Eur Urol 2004;46: 547-554.

11. Hahn RG. Ethanol monitoring of irrigating fluid absorption (review). Eur J Anaesth 1996;13:102-115.

12. Hahn RG. Calculation of irrigant absorption by measurement of breath alcohol level during transurethral resection of the prostate. Br J Urol 1991;68:390-393.

13. Jansen H, Berseus $\mathrm{O}$, Johansson JE. A simple photometric method for determination of blood loss during transurethral surgery. Scand J Urol Nephrol 1978;12:1-5.

14. Rassweiler J, Schulze M, Stock C, et al. Bipolar transurethral resection of the prostate-technical modifications and early clinical experience. Minim Invasive Ther Allied Technol 2007;16:11-21.

15. Mamoulakis C, Ubbink DT, de la Rosette JJ. Bipolar versus monopolar transurethral resection of the prostate: A systematic review and meta-analysis of randomized controlled trials. Eur Urol 2009;56:798-809.

16. Tefekli A, Muslumanoglu AY, Baykal M, et al. A hybrid technique using bipolar energy in transurethral prostate surgery: A prospective, randomized comparison. J Urol 2005;174:1339-1343.

17. Hon NH, Brathwaite D, Hussain $Z$, et al. A prospective, randomized trial comparing conventional transurethral prostate resection with PlasmaKinetic vaporization of the prostate: Physiological changes, early complications and long-term follow up. J Urol 2006;17:205-209.

18. Geavlete B, Multescu R, Dragutescu M, et al. Transurethral resection (TUR) in saline plasma vaporization of the prostate vs standard TUR of the prostate: "the better choice" in benign prostatic hyperplasia? BJU Int 2010;106: 1695-1699.

19. Yoon CJ, Kim JY, Moon KH, et al. Transurethral resection of the prostate with a bipolar tissue management system compared to conventional monopolar resectoscope: Oneyear outcome. Yonsei Med J 2006;47:715-720.

20. Michielsen DP, Coomans D, Peeters I, et al. Conventional monopolar resection or bipolar resection in saline for the management of large ( $>60 \mathrm{~g})$ benign prostatic hyperplasia: An evaluation of morbidity. Minim Invasive Ther Allied Technol 2010;19:207-213.

21. Rassweiler J, Teber D, Kuntz R, et al. Complications of transurethral resection of the prostate (TURP)Incidence, management, and prevention. Eur Urol 2006; 50:969-979.

22. Ahyai SA, Gilling P, Kaplan SA, et al. Meta-analysis of functional outcomes and complications following transurethral procedures for lower urinary tract symptoms resulting from benign prostatic enlargement. Eur Urol 2010;58:384397.

23. Autorino R, Damiano R, Di Lorenzo G, et al. Four-year outcome of a prospective randomised trial comparing bipolar plasmakinetic and monopolar transurethral resection of the prostate. Eur Urol 2009;55:922-929.

24. de Sio M, Autorino R, Quarto G, et al. Gyrus bipolar versus standard monopolar transurethral resection of the prostate: A randomized prospective trial. Urology 2006;67:69-72.

25. Michielsen DP, Coomans D, Braeckman JG, et al. Bipolar transurethral resection in saline: The solution to avoid hyponatraemia and transurethral resection syndrome. Scand J Urol Nephrol 2010;44:228-235.

26. Faul P, Schlenker B, Gratzke C, et al. Clinical and technical aspects of bipolar transurethral prostate resection [Review]. Scand J Urol Nephrol 2008;42:318-323.

27. Williams EL, Hildebrand KL, McCormick SA, et al. The effect of intravenous lactated Ringer's solution versus $0.9 \%$ sodium chloride solution on serum osmolality in human volunteers. Anest Analg 1999;88:999-1003.

28. Wilkes NJ, Woolf R, Mutch M, et al. The effect of balanced versus saline-based hetastarch and crystalloid solutions on acid-base and electrolyte status and gastric mucosal perfusion in elderly surgical patients. Anesth Analg 2001;93:811816.

29. Arieff AI. Fatal postoperative pulmonary edema: Pathogenesis and literature review. Chest 1999;115:1371-1377.

30. Hahn RG, Nennesmo I, Rajs J, et al. Morphological and x-ray microanalytical changes in mammalian tissue after overhydration with irrigation fluids. Eur Urol 1996;29:355-361.

31. Olsson J, Hahn RG. Glycine toxicity after high-dose i.v. infusion of glycine 1.5\% in the mouse. Br J Anaesth 1999;82: 250-254.

32. Michielsen DP, Coomans D. Urethral strictures and bipolar transurethral resection in saline of the prostate: Fact or fiction? J Endourol 2010;24:1333-1337. 
Address correspondence to: Tim Fagerström, M.D. Section of Urology, Södersjukhuset Department of Clinical Science and Education Karolinska Institutet S-118 83 Stockholm Sweden

E-mail: timfagerstrom@hotmail.com; tim.fagerstrom@sodersjukhuset.se

\section{Abbreviations Used}

$\mathrm{BPH}=$ benign prostatic hyperplasia

IPSS $=$ international prostate symptom score QoL $=$ quality of life (bother score)

$\mathrm{TM}=$ timed micturition

TUIP $=$ transurethral incision of the prostate TURis $=$ transurethral resection in saline TURP $=$ transurethral resection of the prostate 
